\title{
Uroflow in murine urethritis
}

\author{
Yuk-Yuen Max Leung ${ }^{1}$, MD, \\ Edward M. Schwarz ${ }^{1,4}$ Ph.D., \\ Christopher R. Silvers ${ }^{2,4}$, \\ Edward M. Messing ${ }^{1} \mathrm{MD}$, \\ Ronald W. Wood ${ }^{1,2,3}$ Ph.D. \\ Departments of Urology ${ }^{1}$, Obstetrics/Gynecology ${ }^{2}$, \\ Neurobiology and Anatomy ${ }^{3}$, Orthopaedics ${ }^{4}$ University of Rochester School of \\ Medicine and Dentistry \\ Rochester NY 14642-8668

\section{Dr. Leung is a Urology Resident.}

Direct correspondence and reprint requests to:

Ronald W. Wood, PhD.

Research Associate Professor

Departments of Obstetrics and Gynecology

University of Rochester School of Medicine

601 Elmwood Ave.

Rochester, NY 14642-8668

Phone: (585) 275-4150

Fax: $\quad$ (585) 244-2209

Email: $\quad$ Ronald Wood@urmc.rochester.eduT

Preprint of a paper to appear in

\section{Urology}

Submitted: December 19, 2003

Revised: March 23, $2004 \quad$ Final Revision: April 9, 2004

A QuickTime movie accompanies this manuscript 1623 Words (Introduction to Acknowledgements, inclusive)

Key Words: Mouse, Uroflow, Urethritis, Obstruction

Running Head: Uroflow in murine urethritis 


\section{Abstract (209 words)}

Objective: Genetically engineered mice may improve understanding of a variety of human bladder diseases. We developed a non-invasive method to measure urinary flow rate in the mouse. This could be useful for the study of bladder outlet obstruction as well as processes affecting detrusor function in the awake animal.

Methods: A metabolic cage without a fecal separation screen was placed above a precision balance that reported the mass of the excreta pan every $100 \mathrm{msec}$. A computational algorithm identified voids suitable for assessment of uroflow from other excretory events. These algorithms were verified by comparison with series of images obtained automatically before and during excretory events. Intraurethral acetic acid was used to induce urethritis and to verify the sensitivity of the measurement technique.

Results: Automatic categorization and characterization of uroflow was successful. Brief exposures of the urethra of the female C57BL6/J mouse to $2 \%$ acetic acid decreased uroflow and increased void duration without a change in void volume.

Conclusions: This method will enable studies of urologic function in mice of differing age, sex, strain, and genetic constitution. Murine urethritis can be differentiated from cystitis, known to be associated with a decrease in void volume. The observed changes are consistent with urethral obstruction induced by local swelling and inflammation. 


\section{Introduction}

Advances in molecular biology have resulted in the ability to develop animal models of voiding dysfunction characterized by deletion or overexpression of messenger RNA in the entire animal or in specific organs. Murine models of voiding function show great promise for the study of voiding dysfunction, but their small size demands the use of special technologies. To study chemical cystitis in the mouse, we placed a diuresis cage with a fecal separation screen and without a collection funnel over an electronic balance $^{1}$. The present report details improvements permitting the collection of more detailed information about each void, including void duration as well as peak and sustained urinary flow rates. A murine urethritis model was developed to study void duration and uroflow in the presence of urethral swelling; this was of particular interest because inducing cystitis with irritant chemicals might also result in urethritis.

Screens for the separation of feces from urine could not be used because they influence the measurement of flow and duration. Thus special computational methods were required to exclude fecal boli, and to identify voids passing cleanly between the floor grids on which the mouse must stand.

\section{Material and Methods}

Uroflow Measurement. Offering a highly preferred sweet solution increased urine output ${ }^{1,2}$. A mouse was placed in a Tecniplast metabolism cage (Nalge-Nunc, Rochester, NY) with a rat grid floor suspended over a $25.4 \mathrm{~cm}$ diameter pan on an electronic balance (PR503DR; Mettler-Toledo, Columbus OH). The cage was rotated 90 degrees to change the orientation of the floor grids in the field of view; this necessitated 
removing some of the plastic from the frame because it obstructed the view of the floor grid. The balance was programmed to generate a record of balance weight 10 times/sec at $1 \mathrm{mg}$ resolution via a serial computer interface (Figure 1). The balance has an internal calibration weight, and is cross-calibrated to external standard weights. A desktop computer logged the entire sequence of records. The balance was calibrated by delivering $2 \mathrm{mls}$ of water to the balance pan using a syringe pump with a $21 \mathrm{G}$ needle on the end of length of rigid plastic tubing attached to the syringe. This was done ten times at individual flow rates ranging from 40 to $80 \mathrm{ul} / \mathrm{sec}$; the flow rates after initial droplet formation approximated the nominal flow rate entered into the computerized syringe pump (PhD 2000, Harvard Apparatus, Holliston, MA). At 40 $\mathrm{ul} / \mathrm{sec}$, a pulsatile pattern became evident as a result of droplet formation at the needle tip.

Electronic balances report when they are unstable, i.e. when a perturbation has occurred on its weighing surface. A separate microprocessor (Basic Stamp 2, Parallax Inc., Rocklin CA) (Figure 1) monitored balance instability and triggered a netcam (Axis 2120, Axis Communications Inc., Chelmsford, MA) focused between the floor grid and the waste pan. The microprocessor displayed the current sample and event numbers, as well as the current mass on the balance on an LCD display in the field of view of the camera. The netcam buffered images continuously and when triggered by $300 \mathrm{msec}$ of balance instability, a series of images that occurred before and during the excretory event were uploaded to an image server via the file transfer protocol (ftp). Since no fecal separation screen was present, the image stream (up to 10/sec) was used to 
validate event discrimination algorithms. Voids passing cleanly between the bars were used to identify the peak flow rate for each void, as well as the median flow rate between 1 and $2 \mathrm{sec}$ after the initiation of each void. Void volume, duration, and frequency could be measured regardless of whether the urine fell between or upon the bars of the floor grid.

Computational algorithms. Balances are intrinsically vibration- and air-current sensitive devices. A simple three-term moving average smoother was applied to the data stream from the balance to eliminate intermittent and extremely small negative transients. The averaging window extended across three samples and stepped with each successive sample. Fecal boli are small and brief events in comparison to voids, and are readily identified computationally (Figure 1, bottom). Voids that pass cleanly between the bars of the floor grid produce a smoothly increasing cumulative weight on the balance pan (Figure 2, left); following an initial peak flow, flow tends to plateau for several hundred milliseconds, and then tail off. Voids that encountered the floor grid resulted in droplet formation that generated a pulsatile pattern (Figure 2, right) that makes the cumulative pattern assume a step-wise appearance. Voids passing cleanly between bars showed low variability between successive samples; however, voids on the bars result in a pulsatile pattern with greater differences between successive voids. The two patterns could be discriminated by computing for each void the ratio of the mean squared successive difference to its variance, rather than to the population variance as in the Von Neumann ratio ${ }^{3,4}$; thresholds were then applied to these ratios, and adjusted until event discrimination corresponded to the image stream. This was facilitated by converting the image stream to a "movie" in which events were colorized 
after the fact by computing the absolute difference between the first image in the sequence and each successive image and then mixing the colorized difference signal with the original gray scale image sequence (video clip; Classification of murine excretory events; http://www.goldjournal.net). The video gives examples of events and the associated balance records. For this image sequence, a $25.4 \times 30.5 \mathrm{~cm}$ acrylic sheet replaced the circular pan, and colorization was restricted to the excretory event by masking.

\section{Induction of urethritis}

Urethritis was induced by introducing the tip of a 24-gauge ball-tipped stainless steel rodent gavage needle into the urethral meatus and infusing $0.8 \mathrm{ml}$ of $2 \%$ acetic acid in 10 minutes under halothane anesthesia. Previous studies ${ }^{5}$ demonstrated that grasping the meatus behind the ball tip is necessary to achieve retrourethral perfusion of the bladder. Overflow was removed in an adsorbent gauze drape.

\section{Statistical Analyses}

Nonparametric one-way analyses of variance by ranks (Kruskal-Wallis tests) were used to determine if an effect was present on any dependent measure during the study period. The nonparametric Mann-Whitney U-test was then used to compare endpoints for days before and after treatment. 


\section{Results}

Urethral irritation prolonged void duration and reduced measures of urinary flow rate. Figure 3 presents boxplots describing the distribution of void durations and flow measures of 14 spaced sessions during which acetic acid was administered intraurethrally twice. Kruskal-Wallis non-parametric one-way analyses of variance by ranks were performed on these measures with days as a categorical variable, and significant effects occurred $(p<.0001)$. The first urethral irritation induction was followed by several days in which the animal only voided a few times in the experimental setting; however, the second administration was followed by sessions with multiple voids.

The large changes observed in these distributions were examined in more detail in the uroflow records (Figure 4) for the day before (day 11) and 3 days after (day 14) the induction of urethritis with acetic acid. The top panels are voids that fell cleanly between the bars of the floor grid on which the animal stood; the bottom panels are voids that encountered the floor grid. Voids impacting the floor grid result in droplet formation (bottom panels); reduction in flow resulted in larger droplets at longer intervals. The void marked "a" in this set of panels went off the edge of the pan in the midst of the void, an observation confirmed with the image sequence data. Excluding the void marked "a", (seen going over the edge of the pan), the baseline data in the top panels was averaged across voids every tenth of a second, and is presented in the right 
panel of this figure, bounded by 2 standard errors of the mean. Comparable means were computed on the third day after the induction of urethritis, and demonstrate a clear difference at each time point except where the functions cross. Void duration was prolonged: the distribution of void durations did not overlap (Figure 4 boxplots). A nonparametric Kruskal-Wallis one-way ANOVA on ranks could not reject the null hypothesis $(p=0.29)$ that no difference occurred on all void durations across the four baseline sessions (days 7,8,10 and 11, top panel of Figure 4) before the second treatment.

A comparison of these endpoints before and after irritant challenge, i.e. days 11 and 14, demonstrated that acetic acid injury of the urethra resulted in a decrease in median peak uroflow, median uroflow from 1 to $2 \mathrm{sec}$ and a prolongation of void duration (all significant at $p<0.01$ by Mann-Whitney $U$ test) (Table 1 ). Void volume did not decrease.

\section{Comment and Conclusions}

This exposure method differentiates murine urethritis and cystitis. Although void volume decreased in our published work on chemically-induced cystitis ${ }^{1}$, void volume did not change in the present urethritis model. The observed changes in flow are almost certainly due to tissue irritation and swelling produced by acid exposure, and are likely causing partial outlet obstruction. Voiding alterations were reversible, and could be reinstated.

Different patterns of functional alteration should enable correlative studies of the underlying anatomic and consequent neurobiologic sequelae, e.g. upregulation of 
neuropeptide expression in anatomically distinct cell bodies identified by retrograde labeling of dorsal root ganglia.

These uroflow methods are likely to be useful for the study of lower urinary tract disorders characterized by obstruction and of pharmacologic or other alterations in detrusor function. This method is fully automated and scalable for large-scale experiments. 


\section{Acknowledgements}

Supported by: Fishbein Family Foundation; Interstitial Cystitis Association; Mae Stone Goode Foundation; Departments of Obstetrics and Gynecology, and of Urology; NIH grant: DK 057679. Dr. Leung is a Urology Resident. 


\section{References}

1. Wood R, Eichel L, Messing EM and Schwarz E: Automated noninvasive measurement of cyclophosphamide-induced changes in murine voiding frequency and volume. J Urol. 165: 653-9., 2001.

2. Valenstein ES, Cox VC and Kakolewski JW: Polydipsia elicited by the synergistic action of a saccharin and glucose solution. Science. 157: 552-4, 1967.

3. Von Neumann J: Distribution of the ratio of the mean square successive difference and the variance. Ann. Math. Stat. 12: 367-395, 1941.

4. Von Neumann J KR, Bellinson HR, Hart BI.: The mean square successive difference. Ann. Math. Stat. 12: 153-162, 1941.

5. Eichel L, Scheidweiler K, Kost J, Shojaie J, Schwarz E, Messing E and Wood R: Assessment of murine bladder permeability with fluorescein: validation with cyclophosphamide and protamine. Urology. 58: 113-8, 2001.

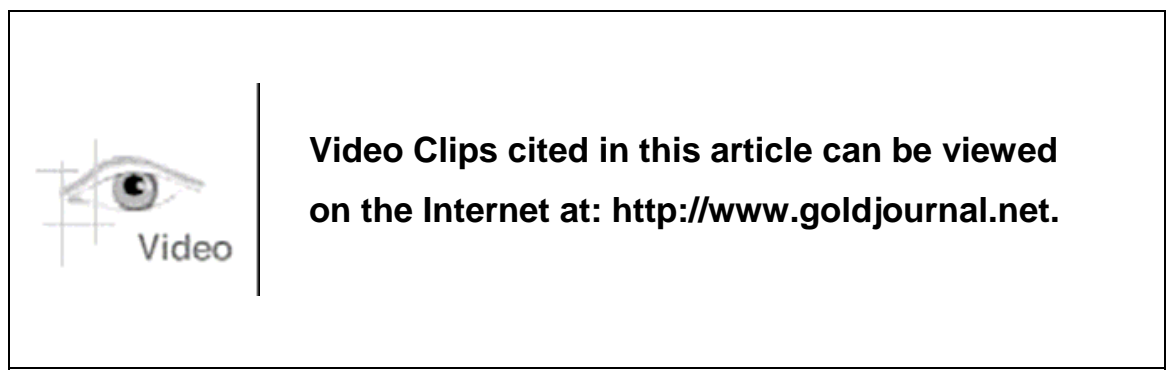


Table 1 Nonparametric statistical evaluation of effects of intraurethral infusion of acetic acid.

\begin{tabular}{|c|c|c|c|c|}
\hline Void Measure & Median Before & Median After & $\underline{U}$ & $\underline{p}$ \\
\hline Duration & 3.8 & 5.35 & $\begin{array}{c}190 \\
n=15,16\end{array}$ & $<=0.01$ \\
\hline Volume & 0.41 & 0.412 & $\begin{array}{c}119 \\
n=15,15\end{array}$ & $>.05$ \\
\hline Peak Flow & 0.15 & 0.112 & $\begin{array}{c}72 \\
n=12,6\end{array}$ & $<=0.01$ \\
\hline $\begin{array}{l}\text { Median Flow } \\
\text { (from } 1 \text { to } 2 \mathrm{sec} \text { ) }\end{array}$ & 0.14 & 0.095 & $\begin{array}{c}72 \\
n=12,6\end{array}$ & $<=0.01$ \\
\hline
\end{tabular}




\section{Figure Legends}

Figure 1. Top: Diagram of system components. Diuresis cage without fecal separation screen is positioned over pan resting on balance. Serial output data stream is monitored by a data-logging computer and by a microprocessor monitoring balance stability. When the balance is unstable for $300 \mathrm{msec}$, the netcam is triggered to transmit a previous image buffer and to continue sending images until the event has terminated. Bottom: Visually confirmed events from an early session. Fecal boli resulted in small brief events in comparison to more prolonged events. Points denote weights obtained when the balance reported stability. Note that the $\sim 60 \mathrm{ul} / \mathrm{sec}$ voids did not differ appreciably in average flow or duration, but display a different pattern suggesting droplet formation.

Figure 2. Representative uroflow (panel A, top) and cumulative volume plot (panel A, bottom) for a void that passed between the bars of the floor grid. Panel B: uroflow and cumulative volume for a void that struck the bars of the floor grid.

Figure 3. Effects of intraurethral acetic acid on void duration, peak uroflow, and the median uroflow derived from one to two seconds after voiding commences. Urethral irritation moved the void duration distribution to longer values, and the uroflow distributions to lower rates. Intraurethral acetic acid was delivered twice and is denoted by vertical lines; dashed lines denote weekends. Void duration (and volume, not shown) estimates were based on all voids; flow measures were derived only for voids passing unobstructed between the floor grids. Fluid intake was reduced for several days after the first acetic acid urethral challenge. Boxplots display the first, second (median) and third quartiles of the event distribution. The tails of the boxplot encompass the range of observations if there are no outliers. If there is a large outlier, i.e. a score greater than the third quartile plus 1.5 times the interquartile range, then the tail extends to the adjacent value, i.e. the largest score less than the outlier criterion. The reverse is true for small outliers.

Figure 4. Effects of intraurethral acetic acid on uroflow in individual sessions. Left two panels present uroflow before and three days after the second acetic acid application, for voids passing through (top panels) or landing on (bottom panels) the floor grids. Void marked "a" was imaged passing off the edge of the balance pan, and was excluded from the calculations of means (right panel). All flow measures were based only on voids passing unobstructed between the floor grids. The control means are bounded by 2 standard errors of the mean. Horizontal boxplots depict the range and quartiles of the duration distributions, which did not overlap. The confidence limits bounding the mean uroflow increased as the number of voids included in the calculation decreased. 


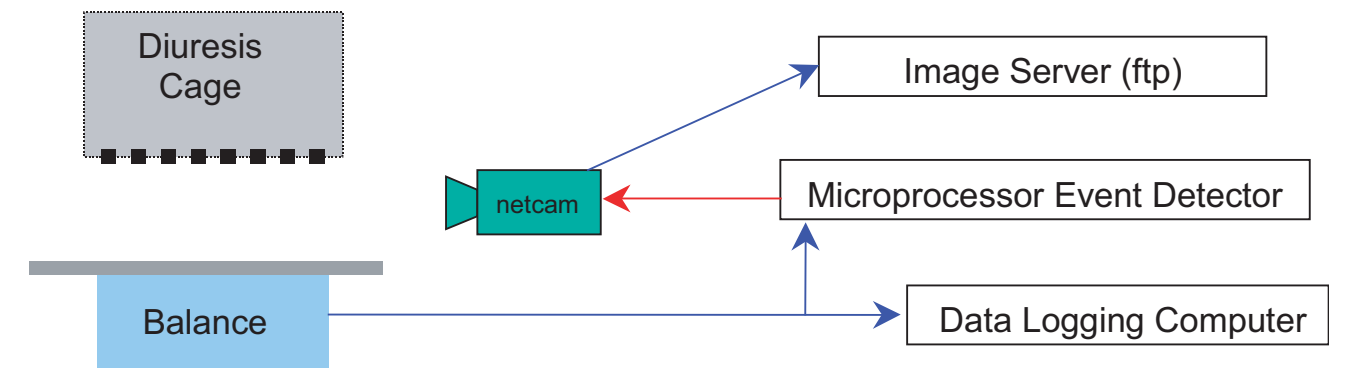

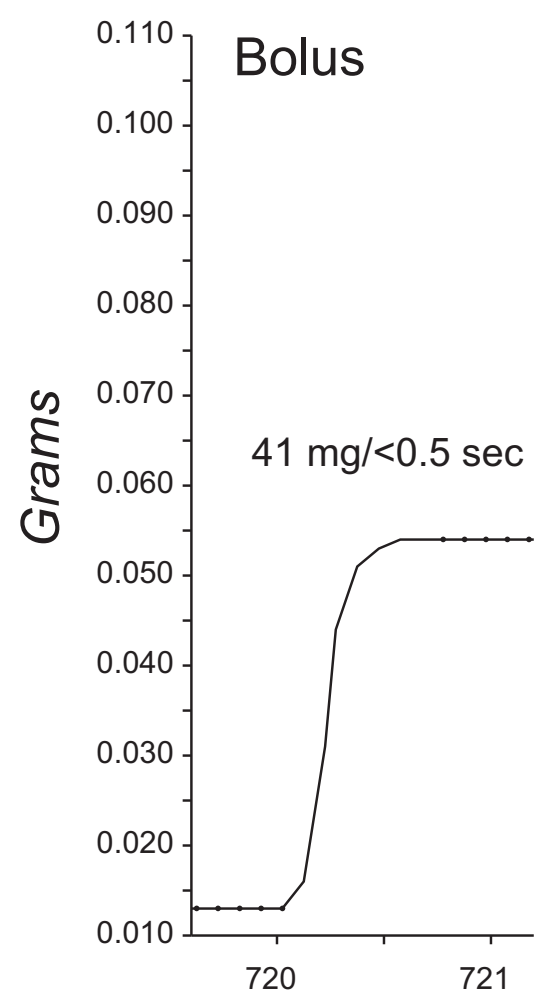

C57BI6 29.8g female
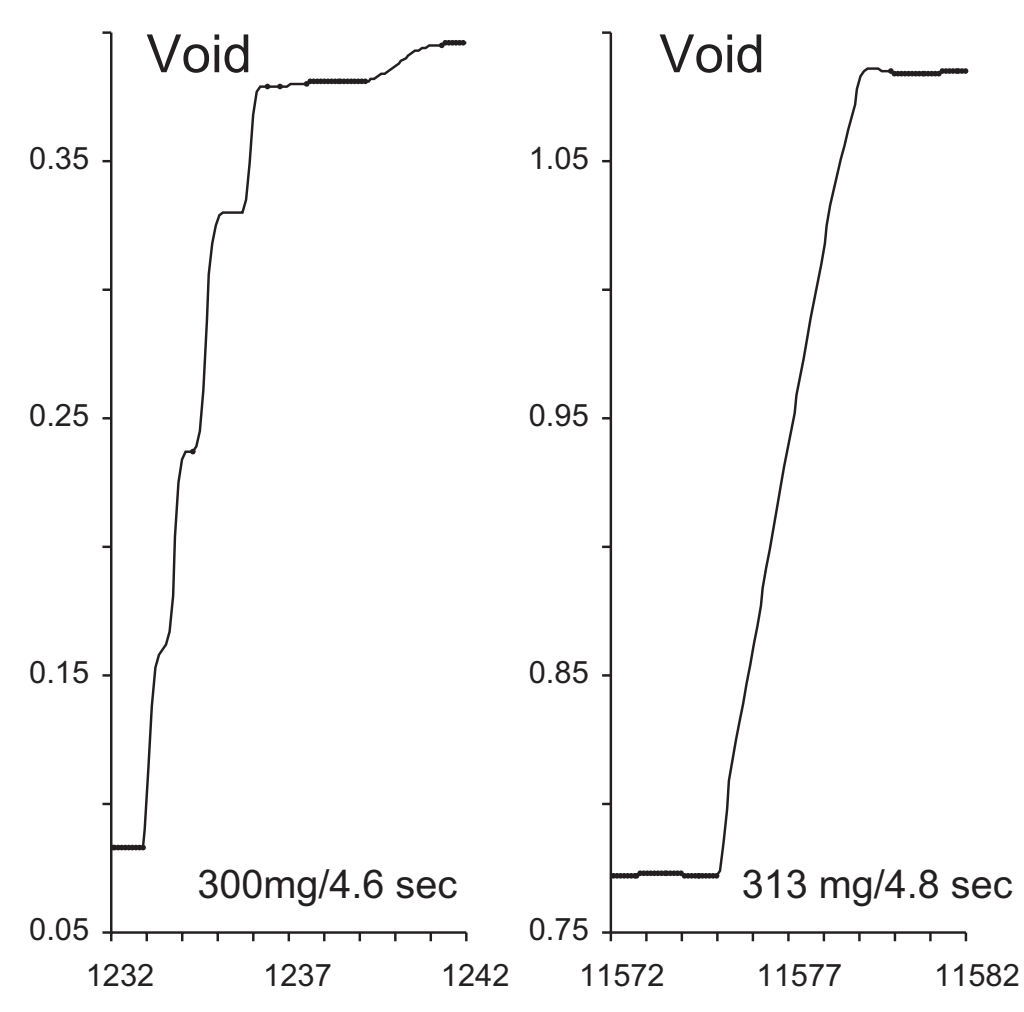

Elapsed seconds

Figure 1. Top: Diagram of system components. Diuresis cage without fecal separation screen is positioned over pan resting on balance. Serial output data stream is monitored by a data-logging computer and by a microprocessor monitoring balance stability. When the balance is unstable for 300 $\mathrm{msec}$, the netcam is triggered to transmit a previous image buffer and to continue sending images until the event has terminated. Bottom: Visually confirmed events from an early session. Fecal boli resulted in small brief events in comparison to more prolonged events. Points denote weights obtained when the balance reported stability. Note that the $\sim 60 \mathrm{ul} / \mathrm{sec}$ voids did not differ appreciably in average flow or duration, but display a different pattern suggesting droplet formation. 

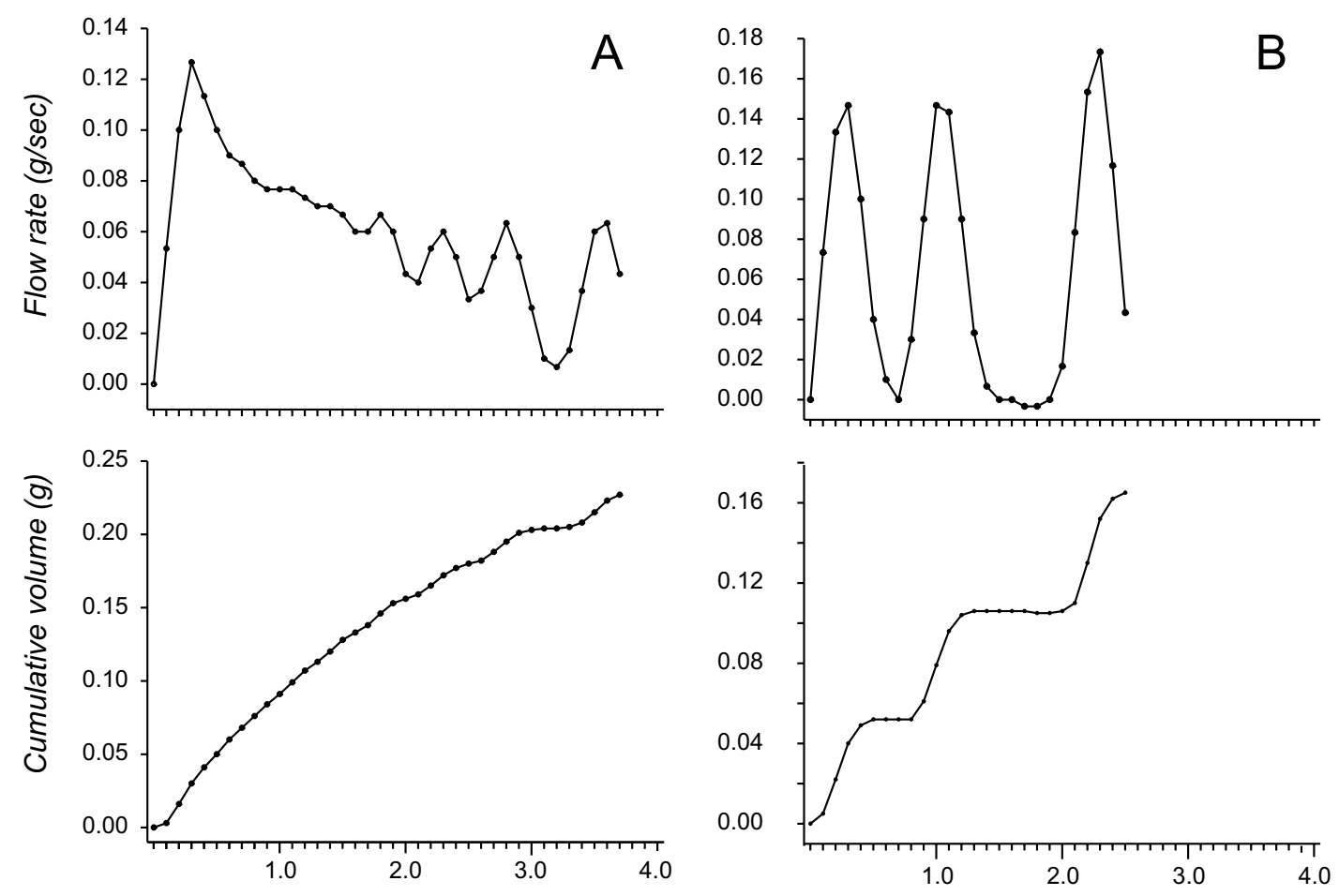

Duration (sec)

Figure 2. Representative uroflow (panel A, top) and cumulative volume plot (panel A, bottom) for a void that passed between the bars of the floor grid. Panel B: uroflow and cumulative volume for a void that struck the bars of the floor grid. 

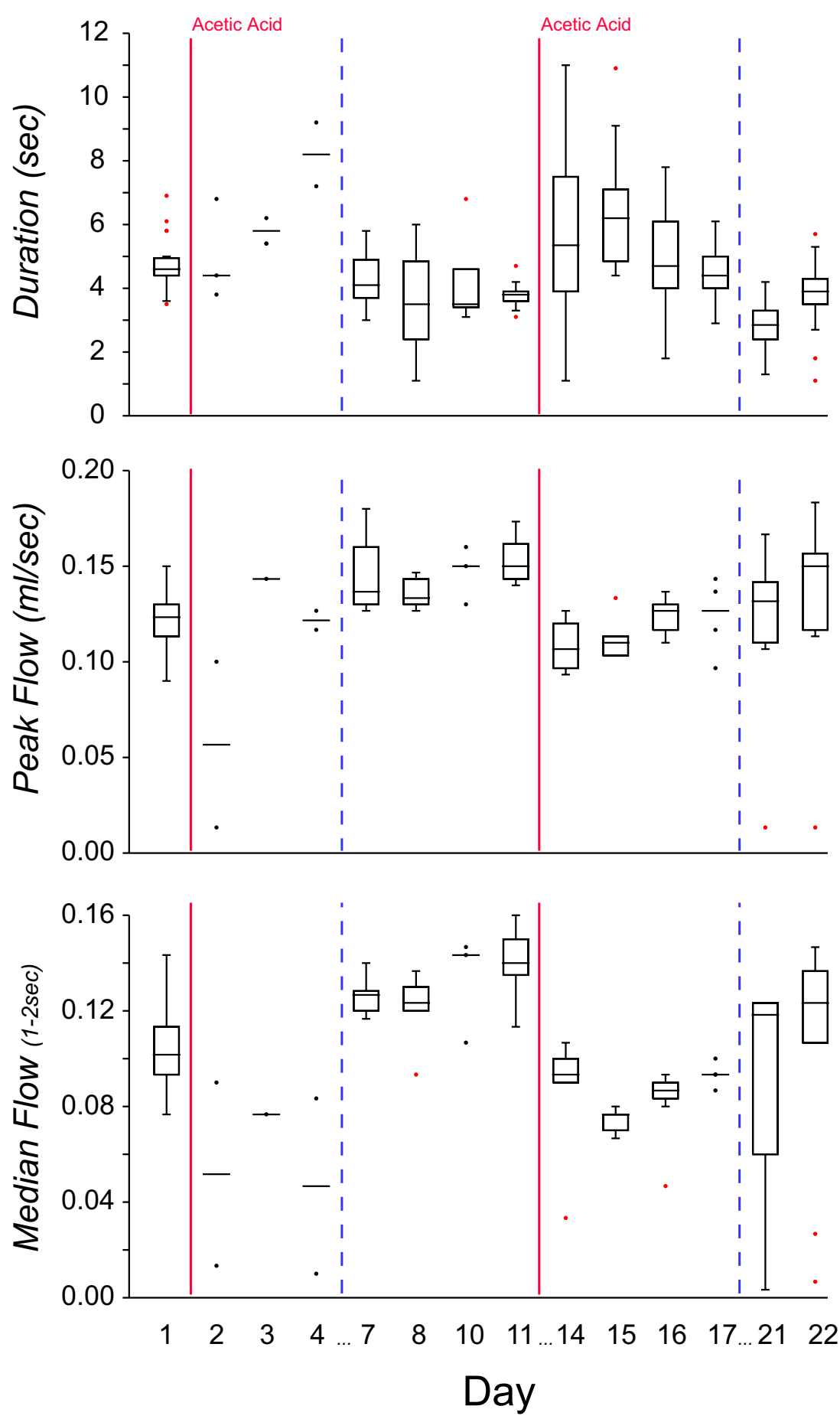

Fig 3. Effects of intraurethral acetic acid on void duration, peak uroflow, and the median uroflow derived from one to two seconds after voiding commences.

Urethral irritation moved the void duration distribution to longer values, and the uroflow distributions to lower rates. Intraurethral acetic acid was delivered twice and is denoted by vertical lines; dashed lines denote weekends.

Void duration (and volume, not shown) estimates were based on all voids; flow measures were derived only for voids passing unobstructed between the floor grids. Fluid intake was reduced for several days after the first acetic acid urethral challenge.

Boxplots display the first, second (median) and third quartiles of the event distribution. The tails of the boxplot encompass the range of observations if there are no outliers. If there is a large outlier, i.e. a score greater than the third quartile plus 1.5 times the interquartile range, then the tail extends to the adjacent value, i.e. the largest score less than the outlier criterion. The reverse is true for small outliers. 

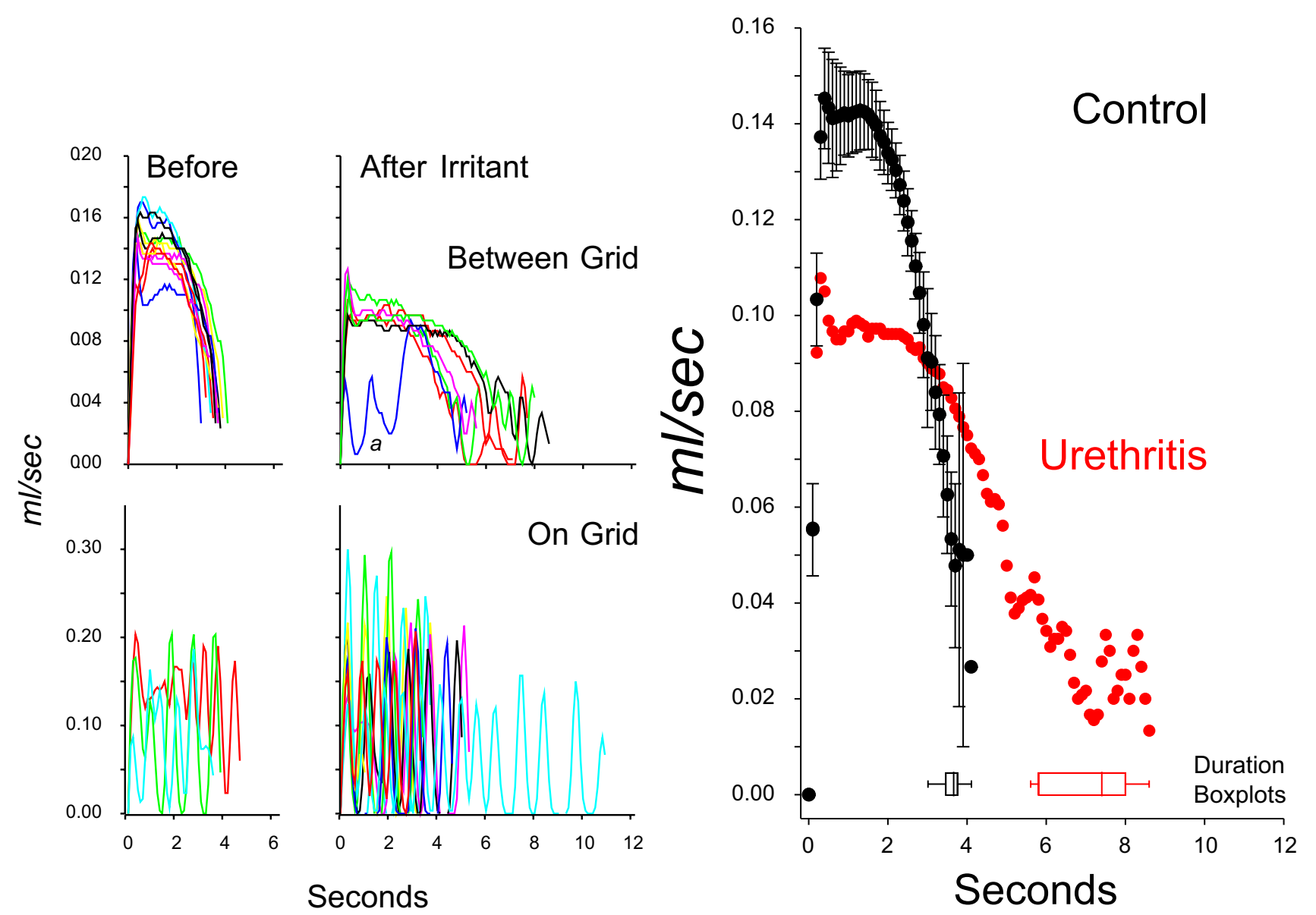

Figure 4. Effects of intraurethral acetic acid on uroflow. Left two panels present uroflow before and three days after the second acid application, for voids passing through (top panels) or landing on (bottom panels) the floor grids. Void marked "a" was imaged passing off the edge of the balance pan, and was excluded from the calculations of means (right panel). All flow measures were based only on voids passing unobstructed between the floor grids. The control means are bounded by 2 standard errors of the mean. Horizontal boxplots depict the range and quartiles of the duration distributions, which did not overlap. The confidence limits bounding the mean uroflow increased as the number of voids included in the calculation decreased. 